OPEN ACCESS

Edited by:

Li-Long Pan,

Fudan University, China

Reviewed by:

Claudio Ferrante

Università degli Studi G. d'Annunzio

Chieti e Pescara, Italy

Luigi Brunetti,

Università degli Studi G. d'Annunzio

Chieti e Pescara, Italy

*Correspondence:

Jianxun Liu

liujx0324@sina.com

Specialty section: This article was submitted to Inflammation Pharmacology,

a section of the journal

Frontiers in Pharmacology

Received: 27 January 2018

Accepted: 30 April 2018

Published: 16 May 2018

Citation:

Zhang Y, Liu J, Yang B, Zheng Y, Yao $M$, Sun $M, X u L$, Lin $C$,

Chang D and Tian F (2018) Ginkgo biloba Extract Inhibits Astrocytic Lipocalin-2 Expression and Alleviates Neuroinflammatory Injury via the JAK2/STAT3 Pathway After

Ischemic Brain Stroke.

Front. Pharmacol. 9:518. doi: 10.3389/fphar.2018.00518

\section{Ginkgo biloba Extract Inhibits Astrocytic Lipocalin-2 Expression and Alleviates Neuroinflammatory Injury via the JAK2/STAT3 Pathway After Ischemic Brain Stroke}

\author{
Yehao Zhang ${ }^{1}$, Jianxun Liu',2*, Bin Yang', Yongqiu Zheng ${ }^{1}$, Mingjiang Yao', \\ Mingqian Sun', Li Xu' ${ }^{1}$, Chengren Lin', Dennis Chang ${ }^{2}$ and Fangze Tian'
}

${ }^{1}$ Beijing Key Laboratory of Pharmacology of Chinese Materia Region, Institute of Basic Medical Sciences of Xiyuan Hospital, China Academy of Chinese Medical Sciences, Beijing, China, ${ }^{2}$ National Institute of Complementary Medicine, Western Sydney University, Penrith, NSW, Australia

Background: Astrogliosis has the potential to lead to harmful effects, namely, neuroinflammation, and to interfere with synapse sprouting. Previous studies have suggested that Lipocalin-2 (LCN2) acts as a key target in regulating the reaction of astrocytes. However, the underlying molecular mechanism is not fully elucidated. In the present study, we examined the neuroprotective and anti-inflammatory effects of Ginkgo biloba extract (EGB), a well-known extract with potential immunoregulatory properties in the nervous system.

Methods: Triphenyltetrazolium chloride staining, hematoxylin-eosin staining, electron microscopy, and neurological assessments were performed in a microsphere-embolized rat model. Human astrocytes exposed to oxygen glucose deprivation (OGD) were used for in vitro experiments. Inflammatory cytokines, multi-labeling immunofluorescence, and Western blotting were used to investigate the molecular mechanisms underlying the EGB-mediated anti-inflammatory effects in vivo and in vitro.

Results: EGB markedly attenuated cerebral infarction and neuronal apoptosis, reduced the inflammatory cytokine level, and alleviated neurological deficiencies in cerebral ischemic rats. After surgery, EGB significantly inhibited astrocyte activation, reduced the phosphorylation of STAT3 and JAK2 and decreased LCN2 expression. In vitro, EGB blocked OGD-induced STAT3 activation and the generation of pro-inflammatory cytokines in human astrocytes, and these effects were significantly enhanced by LCN2 overexpression. EGB downregulated these effects enhanced by LCN2 overexpression.

Conclusion: EGB is demonstrated to mediate neuroinflammation, which protects against ischemic brain injury by inhibiting astrogliosis and suppresses neuroinflammation via the LCN2-JAK2/STAT3 pathway, providing insight into a promising therapeutic strategy for ischemic stroke.

Keywords: cerebral ischemia, neuroinflammation, astrocyte, Lipocalin-2 (LCN2), Ginkgo biloba extract 


\section{INTRODUCTION}

Stroke is the primary reason for mortality worldwide, is responsible for approximately 6 million deaths annually and is considered the key reason for long-term disability (Mendis et al., 2015). Acute cerebral ischemia is caused in the great majority of stroke cases by the occlusion of a supplying arterial vessel (Mozaffarian et al., 2016). Inflammation and immune responses are considered essential for the development of strokes (Fu et al., 2015). The immune system is closely related to crucial events determining the fate of the ischemic brain and the survival of stroke patients. Inflammation may ultimately lead to neuronal cell death by inducing the entry of leukocytes (Lucas et al., 2006), the release of inflammatory cytokines (Lambertsen et al., 2012), and apoptosis (Pettigrew et al., 2008). Some reagents that ameliorate ischemia-induced inflammation have a protective effect, (Zhang et al., 1994; Furuya et al., 2001) suggesting that inflammation may be the crucial mechanism responsible for ischemic brain injury (Chamorro and Hallenbeck, 2006).

Astrocytes respond to attacks on the CNS through a process called astrogliosis (Barres, 2008). Several studies have clarified the capabilities and effects of astrogliosis. Through the release of diverse molecules, reactive astrocytes can have several impacts on nearby cells (Pekny and Nilsson, 2005; Sofroniew, 2009). For example, astrocytes can produce a broad repertoire of proinflammatory and anti-inflammatory cytokines to target tissue damage following trauma, ischemia, infection, degenerative disease, or autoimmune inflammation (Argaw et al., 2012; Zamanian et al., 2012; Kim et al., 2014). Recently, studies have demonstrated that LCN2 is related to a number of CNS injury conditions, such as cerebral ischemia (Jin et al., 2014), excitotoxic injury, stab wound (Chan et al., 2014), medial forebrain bundle transection, and lipopolysaccharide (LPS)induced neuroinflammation (Suk, 2016). Reactive astrogliosis is involved in the pathogenic mechanisms of these disease, and LCN2 expression is induced in reactive astrocytes (Jin et al., 2014). Therefore, astrocytes and LCN2 may be potential molecular therapeutic targets for clinicians to treat inflammatory CNS injuries.

The therapeutic actions of Ginkgo biloba have been wellknown in traditional Chinese medicine for over 5000 years. In 1965, G. biloba leaf extract was first introduced and registered for use in medical practice (DeFeudis, 2003), which commonly used for ailments, including peripheral arterial disease, Alzheimer's disease, short-term memory loss, depression, and anxiety (Kleijnen and Knipschild, 1992; Curtis-Prior et al., 1999). Accordingly, several of these molecular mechanisms have been analyzed in various stroke models. Several studies have demonstrated that EGB has a wide range of therapeutic activities,

\footnotetext{
Abbreviations: CCK8, cell counting kit-8; CNS, central nervous system; CXCL, chemokine (C-X-C motif) ligand; EGB, Ginkgo biloba extract; FBS, fetal bovine serum; GFAP, glial fibrillary acidic protein; HE, hematoxylin-eosin; HPLC, high performance liquid chromatography; IL- $1 \alpha$, interleukin- $1 \alpha$; IL- $1 \beta$, interleukin1 $\beta$; IL-6, interleukin -6; JAK2, Janus kinase 2; LCN2, Lipocalin-2; OGD, oxygen glucose deprivation; POAD, peripheral occlusive arterial disease; STAT3, signal transducer and activator of transcription 3; TNF- $\alpha$, tumor necrosis factor-alpha; TTC, triphenyltetrazolium chloride; UV, ultraviolet.
}

such as mitochondrial function recovery (Baliutyte et al., 2014), antioxidation (Mohamed and Abd El-Moneim, 2017), antiinflammation (Li et al., 2017), and apoptosis inhibition (Wang et al., 2015).

In the present study, we investigated the extent to which EGB can alleviate reactive astrocytes and mediate the repair phase in stroke. We used in vitro and in vivo cerebral ischemia models to investigate the extent that EGB can suppress neuroinflammation, and we investigated possible associated mechanisms. Our results showed that EGB significantly inhibited cerebral ischemia development by downregulating the LCN2activated JAK2/STAT3 signaling pathway and inhibiting astrocyte proliferation. Therefore, our findings indicated a novel role for EGB in the treatment of cerebral ischemia.

\section{MATERIALS AND METHODS}

\section{Microsphere-Embolized Rat Model}

We used male SD (Sprague Dawley) rats with a weight of 220250 g (Beijing Vital River Laboratory Animal Technology Co., Ltd., Beijing, China). All the procedures and ethics guidelines were approved by the Committee for Experimental Animal Use and Care of the Chinese Academy of Medical Sciences, China. Efforts were made to minimize the number of animals used and their suffering. The rats were housed at a temperature and humidity of $23 \pm 1^{\circ} \mathrm{C}$ and $55 \pm 5 \%$, respectively, with a 12 -h light-dark cycle. Rats had unrestricted access to food and water.

All 50 adult SD rats were randomly allocated into five groups. Microsphere-induced cerebral embolism was conducted with a previously described method (Moriyama et al., 2013). After anesthetization with $40 \mathrm{mg} / \mathrm{kg}$ chloral hydrate, the common carotid artery and right external carotid of the rats were temporarily occluded with vascular clamps ( $n=10$ per group, 5 groups). A 2-mL syringe was then inserted into the right internal carotid. Microspheres (106-212 $\mu \mathrm{m}$ in diameter, UVPMS-BY2, Cospheric, United States) were suspended in rat serum with $1 \mathrm{mg} / \mathrm{ml}$, and $0.2 \mathrm{~mL}$ of this suspension was injected into the right internal carotid artery. After the injection, the vascular clamps occluding the right external and common carotid arteries were released, and then, the puncture wound was closed with suture strings. Blood flow to the brain by the right external and common carotid arteries recommenced after 2-3 s. Rats in the sham group were injected with the same volume of rat serum but without microspheres.

\section{Drug Administration}

Ginkgo biloba extract were provided in-kind by Shineway Pharmaceutical Group (Shijiazhuang, China), it contains total ginkgo flavone-glycosides from G. biloba. The quantitative analysis results showed that it contained $49 \%$ of total flavones (UV), $28.7 \%$ of sum of glycosides with aglycone of quercetin, isorhamnetin and kaempferol (HPLC-UV), $11.6 \%$ of sum of gingkolide A, B, C and bilobalide with $3.3 \%$ of gingkolide A (HPLC-UV) among them in gingko extract (Zhang et al., 2018). The EGB preparations were manufactured in a Good Manufacturing Practice certified facility. EGB was injected into 
the duodenum of the rats after cerebral ischemia surgery at doses of 7.5 and $15 \mathrm{mg} / \mathrm{kg}$. Then EGB was intragastrically administrated at $24 \mathrm{~h}$ and $48 \mathrm{~h}$ after surgery. Rats in the sham group were administered the same volume of saline.

\section{Evaluation of Neurological Deficits}

Scores pertaining to the neurological deficits of the rats were calculated for each group after $2 \mathrm{~h}$ of ischemia and $24 \mathrm{~h}$. These scores were conducted based on a five-point scale adapted from a previous publication (Bederson et al., 1986). Specifically, if a rat demonstrated normal spontaneous movements (no neurological deficit), they were assigned a score of 0 ; if a rat was unable to fully extend its right paw, they were assigned a score of 1 ; if a rat circled in a clockwise motion, they were assigned a score of 2 ; if a rat fell to the right, they were assigned a score of 3; if a rat was unable to walk, they were assigned a score of 4 . All neurological assessments were conducted by a researcher blinded to all groups.

\section{Measurement of Cerebral Infarction}

Forty-eight hours after cerebral ischemia, $40 \mathrm{mg} / \mathrm{kg}$ chloral hydrate was administered to anesthetize the rats. Before decapitation, blood serum was collected using the abdominal aorta method. The brain was then detached, and 1-mm-thick coronal slices were acquired and fixed in prewarmed $2 \%$ TTC for $10 \mathrm{~min}$. Finally, the slices were fixed in $10 \%$ paraformaldehyde for $30 \mathrm{~min}$.

\section{Electron Microscopy}

At $48 \mathrm{~h}$ after cerebral ischemia, cerebral cortex slices measuring 1 cubic millimeter were crafted into square pieces with a vertex at the center of the ischemic area comprising part of the penumbra and undamaged area. These pieces were fixed for $2 \mathrm{~h}$ with $2.5 \%$ glutaraldehyde in PBS at room temperature. The pieces were washed with PBS, incubated for $1 \mathrm{~h}$ in PBS solution containing $1 \% \mathrm{OsO}_{4}$, dehydrated with ethanol, contrast-stained with $1 \%$ uranyl acetate, and embedded in EPON resin. Ultrathin sections were prepped and observed using the ultramicrotome Leica EM UC6 and a transmission electron microscope HITACHI H-7500 (HITACHI, Japan), respectively.

\section{Human Astrocyte Culture and Transfection}

Astrocytes were purchased from ScienCell Research Laboratories (CA, United States). In this study, cells were grown in Normal astrocyte media (AM) (Sicencell, United States) containing 10\% FBS (Gibco, United States), 1\% l-glutamine, and 1\% penicillinstreptomycin in a humidified atmosphere of $5 \% \mathrm{CO}_{2}$ at $37^{\circ} \mathrm{C}$. The expression vector of the LCN2 gene (EX-m0282-Lv201) and the vector control (EX-NEG-Lv201) were obtained from GeneCopoeia (Rockville, United States). After cells reached 70\% confluence, the astrocytes were stably transfected using $7.5 \mu \mathrm{l} / \mathrm{ml}$ lentiviral vector (LPP-m0282-Lv201-100) from GeneCopoeia (Rockville, United States) for $24 \mathrm{~h}$. Then, the cells were given normal astrocyte media (AM) (Sicencell, United States) containing 10\% FBS (Gibco, United States) without lentiviral vector for $48 \mathrm{~h}$.

\section{Oxygen Glucose Deprivation (OGD) Management and Treatment}

After purification and transfection, human astrocytes were cultured in an incubator with premixed gas $\left(1 \% \mathrm{O}_{2}, 94 \% \mathrm{~N}_{2}, 5 \%\right.$ $\mathrm{CO}_{2}$ ) for $6 \mathrm{~h}$ using deoxygenated DMEM without glucose or FBS. After $6 \mathrm{~h}$, the cells were given normal AM containing 10\% FBS and were transferred to a $\mathrm{CO}_{2}$ incubator with $95 \%$ air and $5 \%$ $\mathrm{CO}_{2}$ environment for $12 \mathrm{~h}$. The cells in the control group were simultaneously cultured with normal AM and 10\% FBS. During OGD induction, astrocytes were treated by $2.5,5$, or $10 \mathrm{mg} / \mathrm{L}$ of EGB for $6 \mathrm{~h}$.

\section{Western Blotting}

The protein from tissues containing ischemic penumbra were harvested at 24, $48 \mathrm{~h}$ after cerebral ischemia was extracted by RIPA buffer (Beyotime Biotechnology, Shanghai, China) and was mixed with protease and phosphatase inhibitor cocktails (MCE, NJ, United States). The protein concentration was determined using a protein assay solution (Bio-Rad). Identical quantities of protein were denatured using protein loading buffer, loaded onto $10 \%$ SDS-PAGE gels, and transferred to polyvinylidene difluoride (PVDF) membranes by electroblotting. The PVDF membranes were blocked by $5 \%$ bovine serum albumin (BSA) in TBST buffer for $1 \mathrm{~h}$ and were incubated overnight at $4^{\circ} \mathrm{C}$ using the following antibodies: LCN2 (Abcam, 1:2000 dilution), GFAP (Proteintech, 1:2000), p-JAK2 (Tyr1007/1008) (Abcam, 1:1000), JAK2 (Abcam, 1:2000), p-STAT3 (Tyr705) (Cell Signaling Technology, 1:2000), STAT3 (Cell Signaling Technology, 1:2000), and $\beta$-actin (Sigma, 1:5000). Reactive bands were detected using ECL detection reagent (Thermo Fisher Scientific, MA, United States) following the manufacturer's instructions. The protocols for cell culture experiments were the same as those described above.

\section{Immunofluorescence Analysis and Hematoxylin-Eosin (HE) Staining}

At $48 \mathrm{~h}$ after cerebral ischemia, paraffin-embedded segments were used to evaluate the expression of GFAP, LCN2, p-STAT3, and p-JAK2 according to immunohistochemical protocols $(n=5$ per group). Brain tissues were fixed in $4 \%$ paraformaldehyde in PBS (0.01 M, pH 7.4) for $24 \mathrm{~h}$ at $4^{\circ} \mathrm{C}$, dehydrated in a graded series of alcohols, and embedded in paraffin. Brain tissues were cut into 5- $\mu$ m-thick sections using a Leica ${ }^{\circledR}$ RM1850 rotary microtome (Leica Microsystems, Germany). To eradicate endogenous peroxidase activity, the sections were incubated in $3 \% \mathrm{H}_{2} \mathrm{O}_{2}$ and $3 \%$ normal goat serum. The sections were then incubated for $1 \mathrm{~h}$ at $37^{\circ} \mathrm{C}$ using the following rabbit polyclonal antibodies: LCN2 (Abcam, 1:200), GFAP (Proteintech, 1:200), p-JAK2 (Tyr1007/1008) (Abcam, 1:50), JAK2 (Abcam, 1:200), p-STAT3 (Tyr705) (Cell Signaling Technology, 1:100), and STAT3 (Cell Signaling Technology, 1:200). The sections were rinsed with PBS and incubated with secondary antibodies (Cell Signaling Technology, 1:100) at room temperature for $45 \mathrm{~min}$; then, the sections were developed by diaminobenzidine and counterstained with hematoxylin. Secondary antibodies, biotinylated conjugates, and diaminobenzidine were obtained 
using a streptavidin-peroxidase kit, and the resulting signals were imaged. Brain sections were counterstained with Hoechst 33342 $(10 \mu \mathrm{g} / \mathrm{mL})$ for $15 \mathrm{~min}$ to distinguish the nucleus. To obtain color images, a $20 \times$ laser scanning confocal microscope (Olympus FV1200, Tokyo, Japan) was utilized.

\section{Determination of Chemokine/Cytokine Expression in Brain Extracts, Serum Samples and Astrocytes}

The chemokine and cytokine concentrations in brain hemisphere extracts, serum samples and astrocytes conditioned in medium were quantified by the EMD Millipore's MILLIPLEX ${ }^{\circledR}$ MAP Rat Cytokine/Chemokine Magnetic Bead assay in compliance with the manufacturer's instructions. The chemokine and cytokine concentrations from tissues containing ischemic penumbra were harvested at $48 \mathrm{~h}$ after cerebral ischemia was extracted by EMD Millipore's buffer. Blood serum was collected using the abdominal aorta method. The chemokine and cytokine concentrations in astrocytes conditioned in medium were harvested after OGD induction. The cytokines TNF $\alpha$, IL- $1 \alpha$, IL- $1 \beta$, and IL- 6 and the chemokine CXCL10 (IP-10) were analyzed. The median fluorescence intensity was assayed on a FLEXMAP $3 \mathrm{D}^{\mathrm{TM}}$ system. A five-parameter logistic method was performed to approximate the cytokine and chemokine concentrations in both the brain homogenates and serums samples.

\section{Statistical Analyses}

All statistical data were analyzed using GraphPad Prism software (San Diego, CA, United States). The results are expressed as the means \pm SEM. Each experiment was performed in triplicate. Multiple group comparisons were analyzed using one-way analysis of variance (ANOVA). Student's $t$-test was conducted to analyze intergroup comparisons. For neurological studies, twoway ANOVA was conducted for intergroup pairwise comparisons as a function of time. $P$-values of $(P<0.05)$ were considered statistically significant.

\section{RESULTS}

\section{EGB Protected Against Cerebral Ischemia-Induced Brain Injury}

Ginkgo biloba extract was injected into the duodenum of rats after the occurrence of cerebral ischemia. Brain infarct volumes were determined by TTC assay $48 \mathrm{~h}$ after surgery. EGB dose-dependently reduced brain infarct volume. Accordingly, the neurological deficit scores mirrored the results of infarct volume, indicating the protective effects of EGB. These data suggested a neuroprotective role of EGB in reperfusion after ischemia. Compared to the cerebral ischemia group, the EGB7.5 (7.5 mg/kg, daily) and EGB-15 (15 mg/kg, daily) groups had a significant reduction in neurological deficit scores $(P<0.05)$. Moreover, the neurological deficit score in the EGB15 group was less than that in the EGB-7.5 group. Figure 1C shows the neurological deficit scores for all groups at 2 and
$24 \mathrm{~h}$ after surgery and the occurrence of cerebral ischemia, respectively.

Figures 1A,B show the infarct volume of all groups at $48 \mathrm{~h}$ after cerebral ischemia occurred. In the cerebral ischemia group, extensive lesions were observed in the striatum, hippocampus, and cortex. Consistent with the neurological deficit scores, the group treated with EGB $(15 \mathrm{mg} / \mathrm{kg}$, daily) had reduced infarct volume and showed a significant therapeutic effect compared with the cerebral ischemia group.

Hematoxylin-Eosin staining was observed to identify the histological changes of brain neurons for all groups (Figure 1D). Cortex sections and hippocampal regions stained with $\mathrm{HE}$ presented with neuronal loss and signs of cerebral edema, and swollen cells were observed in the ipsilateral frontal cortex. After surgery, several apoptotic neurons were observed with karyopyknosis, cell gaps and debris. EGB (15 mg/kg, daily) significantly alleviated the symptoms of apoptosis in a dosedependent manner. Therefore, these results indicated that $15 \mathrm{mg} / \mathrm{kg}$ EGB can reduce brain injury caused by cerebral ischemia.

\section{Ultrastructural Changes Associated With EGB Treatment}

Electron microscopy studies were performed to generate a detailed evaluation of astrocyte phenotypes in the peri-infarct area following a stroke (Figure 2). The cytoplasm of the astrocytes was occasionally observed to be completely empty, with dispersed organelles and fibrils (Figures 2A-E). In the model group, swollen astrocytes adhered to the damaged area, which indicated activation, as evidenced by a significant amount of chromatin condensation on top of the nuclear membrane and a large nucleolus. Activated astrocytes and two-cell fusion were also observed (Figure 2C). These astrocytes were not observed in the control group. Finally, EGB treatment $(15 \mathrm{mg} / \mathrm{kg}$, daily) reduced the damage to brain tissue elements in a dose-dependent manner, and this tissue had a more viable appearance.

\section{Effect of EGB on Inflammatory Markers}

A significant difference in cytokine levels was observed after surgery. For example, at $48 \mathrm{~h}$, pro-inflammatory IL- $1 \alpha$, IL- $1 \beta$ and chemokine CXCL10 (IP-10) were noted in the ipsilateral hemisphere of the model group but were not observed in the control group. The degree of statistical significance was calculated at $p<0.001$ for IL- $1 \alpha, p<0.001$ for IL-1 $\beta$, and $p<0.01$ for the chemokine CXCL10. However, EGB significantly reduced these cytokine levels compared with those of the cerebral ischemia group ( $p<0.05$, EGB-15 group vs. model group; $p<0.01, \mathrm{EGB}-15$ group vs. model group) (Figures 3A-C). Additionally, identical results were found in the rats' serum. The levels of IL- $1 \alpha$, IL-1 $\beta$ and CXCL10 in the model group were markedly higher than those of the sham group $(p<0.05)$ (Figures 3D-F). Similarly, the EGB-15 group presented with reduced cytokine levels compared with the cerebral ischemia group ( $p<0.01$, EGB-15 group vs. model group). 

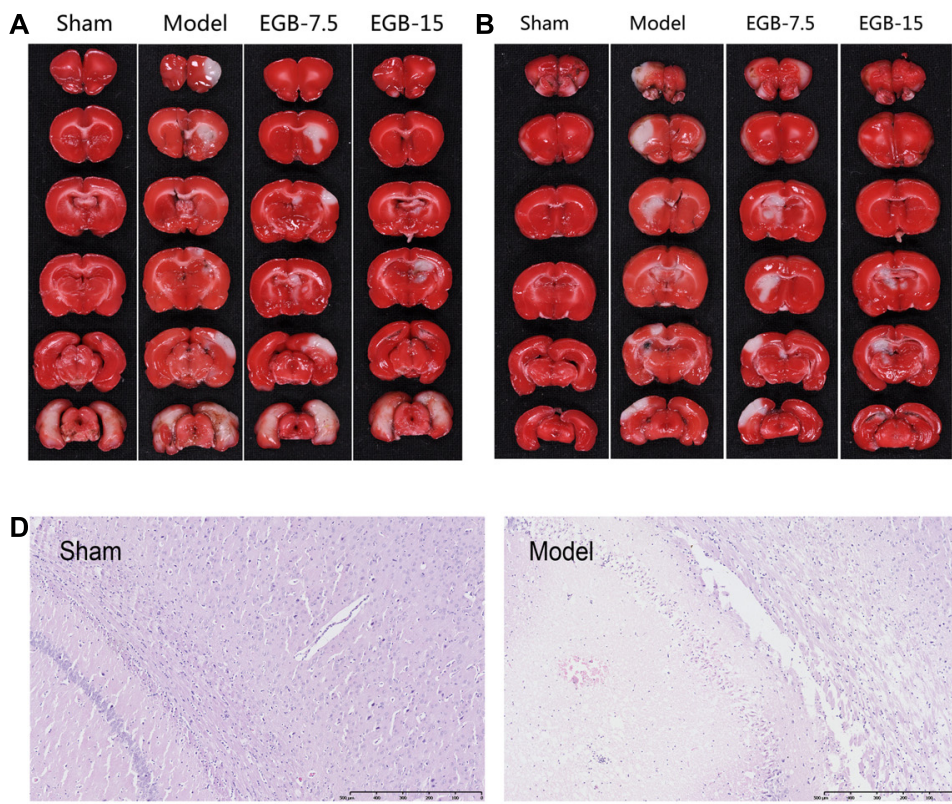

EGB-7.5
Model
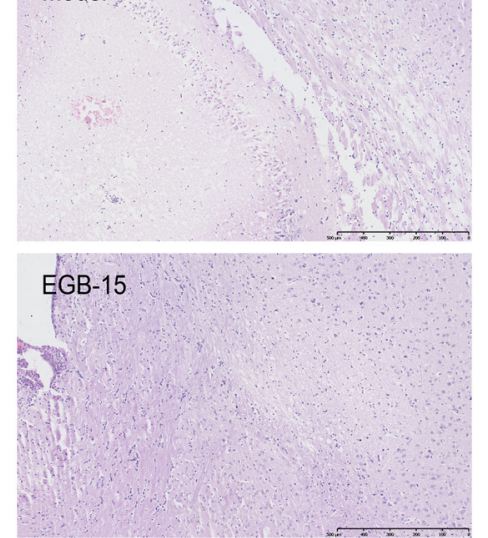

C
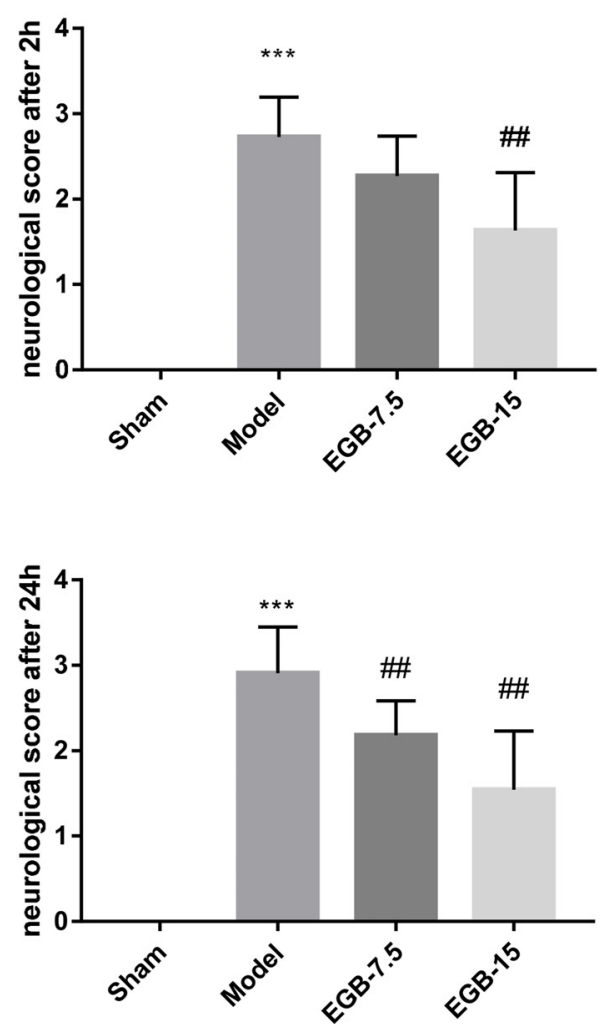

FIGURE 1 | EGB treatment reduced ischemic infarct volume in the cerebral ischemia model. (A,B) Cerebral infarct volume was assessed via $\Pi T C$ staining $48 \mathrm{~h}$ after cerebral ischemia. Neurological score (C) of rats after cerebral ischemia were assessed using a five-point scale system. Data are expressed as mean \pm SEM ( $n=10)$. ${ }^{* * *} p<0.01$ vs. sham group; \#\# $\quad 0.01$ vs. the model groups. The ischemic penumbra area in the box (D) was assessed for neuronal apoptosis using HE staining.

\section{EGB Alleviates LCN2 Up-Regulation in a Rodent Model of Cerebral Ischemia}

Immunofluorescence staining and Western blot analysis revealed a significant increase in brain LCN2 protein levels after cerebral ischemia. An EGB injection into the duodenum reduced cerebral ischemia-induced LCN2 levels. To determine the cellular localization of LCN2 protein in the hippocampus of cerebral ischemic rats, double-immunofluorescence staining for LCN2 and GFAP was performed in brain sections after cerebral ischemia. LCN2 protein expression was localized to within GFAP-positive astrocytes. Consistent with the results shown in Figure 4, the expression of LCN2 was induced, and the number of astrocytes in the cortex was increased in rats with cerebral ischemia compared with the sham-operated rats. However, EGB significantly reduced GFAP levels. The effects of EGB on astrocyte activation, LCN2, p-STAT3, and p-JAK2 expression in the ipsilateral peri-infarct cortical area $(-1.7$ to $-1.9 \mathrm{~mm}$ from the bregma) following cerebral ischemia were captured with a laser confocal microscope (Olympus FV1200, Japan). To determine the potential role of EGB and LCN2 on neuroinflammation caused by ischemic injury, astrocyte activation status was assessed. EGB treatment $(15 \mathrm{mg} / \mathrm{kg})$ suppressed the activation of astrocytes in the ischemic hemisphere, as suggested by the reduced GFAP levels detected by immunofluorescence staining and Western blotting ( $p<0.05$, EGB group vs. model group). In addition, double-immunofluorescence staining also demonstrated that astrocytic p-STAT3 and p-JAK2 levels were markedly increased after cerebral ischemia but were diminished by EGB treatment (Figures $4 \mathrm{~A}-\mathrm{C}$ ). The expression of GFAP, LCN2, p-STAT3, and p-JAK2 was determined using Western blot (Figures 4D-H). LCN2 was observed to increase in the cerebral ischemia group compared to the sham group $(p<0.05)$. Finally, EGB markedly downregulated LCN2 expression in the cerebral ischemia group compared to the sham group $(p<0.01)$. A similar pattern of expression was noted for p-STAT3 and p-JAK2.

\section{EGB Attenuates OGD-Induced Injury in Astrocytes}

CCK8 assays revealed that cell viability was significantly reduced by OGD-induced injury. EGB $(50 \sim 200 \mathrm{mg} / \mathrm{L})$ was observed to have a dose-dependent toxic effect on the viability (CCK8 assay) of the human astrocytes. However, treatment with EGB $(12.5 \sim 50 \mathrm{mg} / \mathrm{L})$ attenuate the OGD-induced decrease in cellular activity. Finally, the pesticide effect was noted in the $2.5 \mathrm{mg} / \mathrm{L}$ and $5 \mathrm{mg} / \mathrm{L}$ EGB groups ( $p<0.05$, vs. OGD group); therefore, 


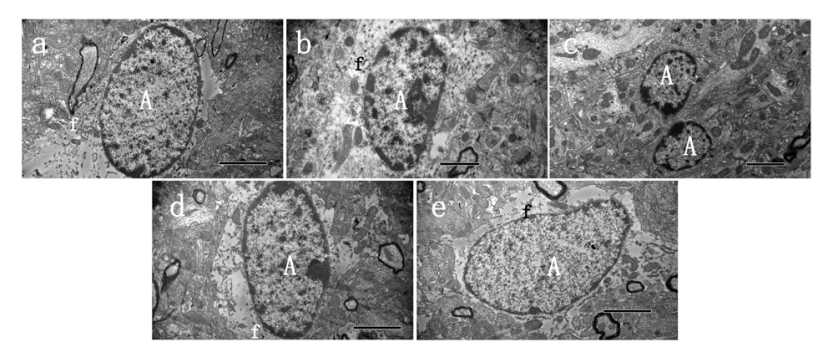

FIGURE 2 | Ultrastructural characteristics of astrocytes $48 \mathrm{~h}$ after cerebral ischemia. Representative transmission electron microscopy images of astrocytes in the peri-infarct area of stroke, at $48 \mathrm{~h}$ after cerebral ischemia, in three groups of animals. (A) Normal astrocyte was observed in sham group, (B) Swollen activated perineuronal and perivascular astrocytes were seen in model groups, which demonstrated activation as evidenced by a significant amount of chromatin condensation atop the nuclear membrane and large nucleolus. The astrocytes were characterized by dispersed residual organelles and swollen mitochondria. Astrocyte fusion was often observed (C). (D,E) Less damage was observed in the EGB treatment group. A astrocyte, f-fibrils. Bars: A,C,D,E $2 \mu \mathrm{m}$; B $1 \mu \mathrm{m}$.

$5 \mathrm{mg} / \mathrm{L}$ EGB was administered in the following in vitro tests (Supplementary Figure S1).

To determine the role of LCN2 in the anti-inflammatory effects of EGB, LCN2 was overexpressed in human astrocytes. The cells were incubated under OGD settings to simulate an ischemic model in vitro. After OGD induction, the EGB treatment group had significantly reduced levels of the proinflammatory cytokines IL-6, IL-1 $\beta$, and CXCL10 compared to the model group $(p<0.05)$. This effect was enhanced by LCN2 overexpression ( $p<0.05$, vs. control group) (Figures 5A-C).

A change in astrocyte morphology was determined by GFAP staining (Figures 6A-C). OGD-induced astrocyte activation was confirmed by lengthened cellular protuberances, and this effect was enhanced by LCN2 overexpression. EGB alleviated the activation of astrocytes after OGD induction. To investigate the downstream anti-inflammatory targets of EGB, the expression levels of LCN2, p-JAK2, and p-STAT3 were examined in human astrocytes. Compared to the control group, the OGDstimulated model group presented with dramatically increased levels of GFAP, LCN2, p-JAK2, and p-STAT3 $(p<0.05$, $p<0.01$ ) (Figures 6A-H). These effects were enhanced by LCN2 overexpression, as evidenced by immunofluorescence staining and Western blotting (Figure 6). EGB was observed to markedly reduce GFAP, LCN2, p-JAK2, and p-STAT3 levels after OGD stimulation $(p<0.05, p<0.01)$. Interestingly, in human astrocytes, EGB significantly decreased the level of LCN2 overexpression and suppressed the phosphorylation of STAT3 and JAK2 after OGD. No differences between the control group and the EGB-treated control group were observed.

\section{DISCUSSION}

In this study, a novel signaling pathway involving LCN2 for the EGB-mediated dysfunction of reactive astrocyte properties in ischemia was reported. EGB markedly reduced

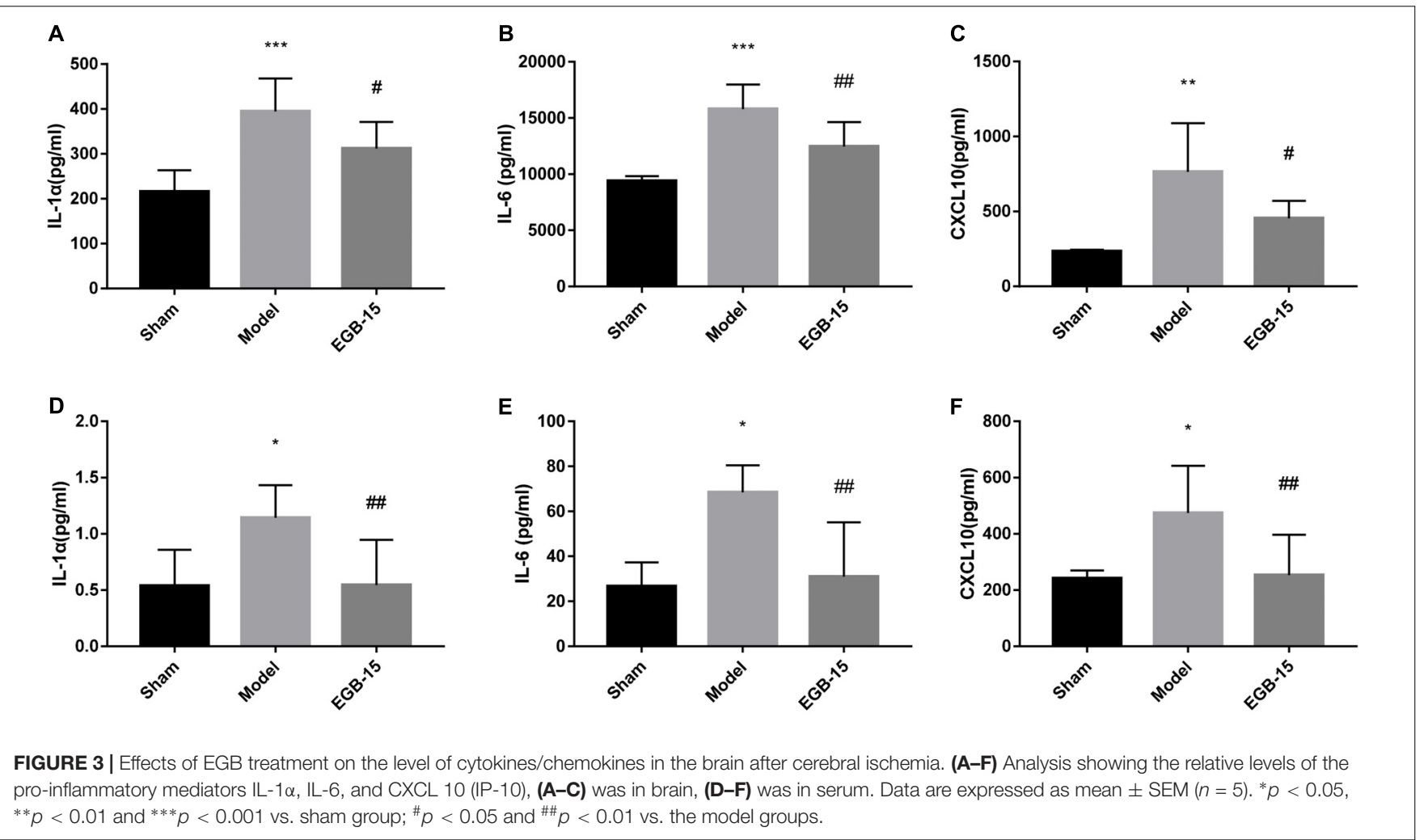



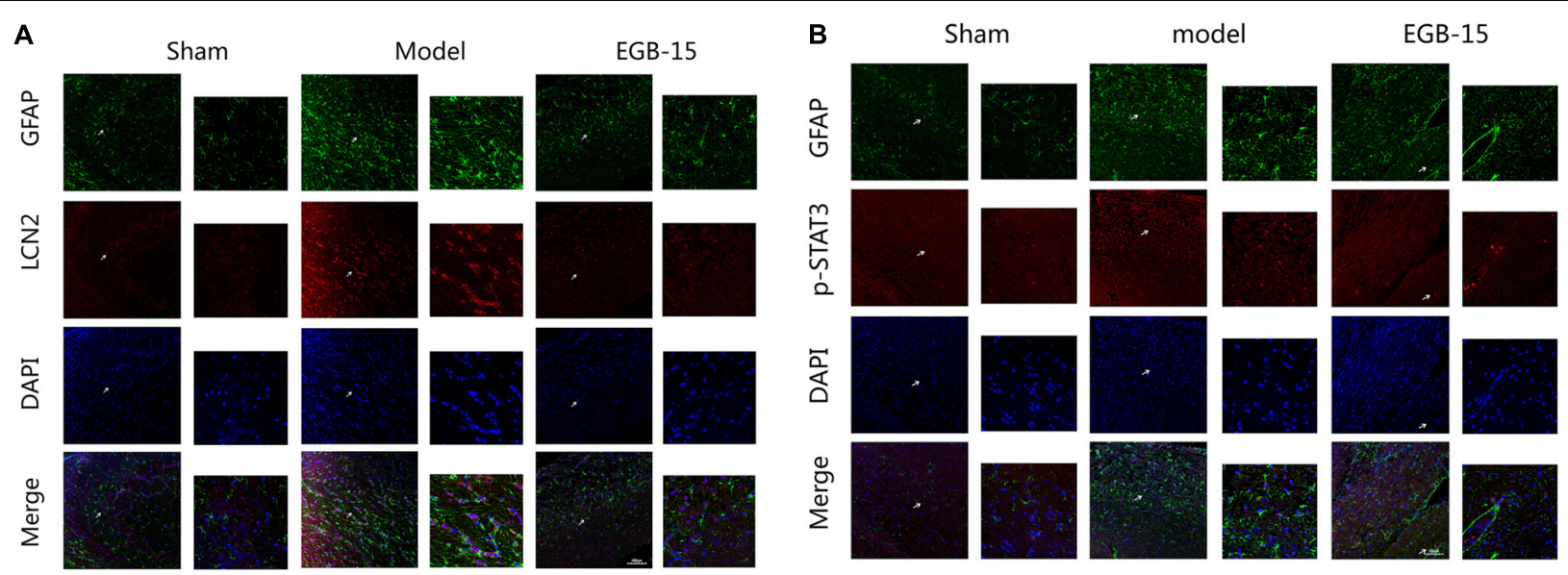

C

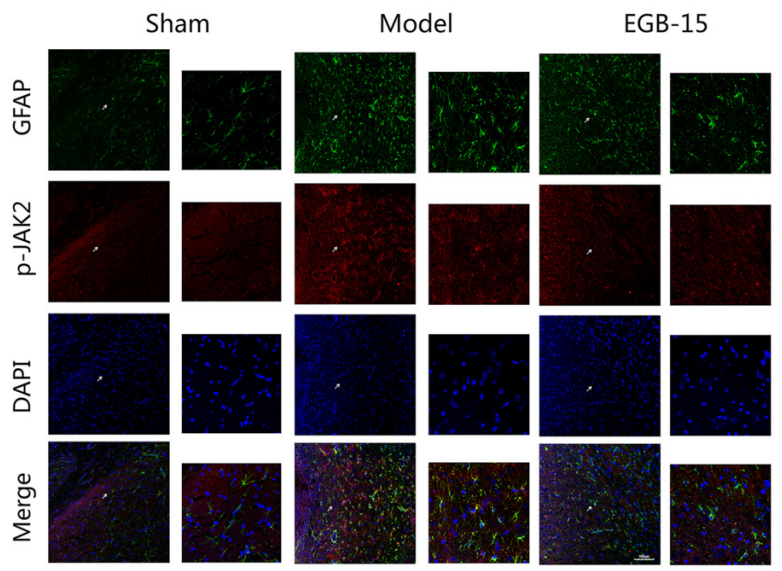

D
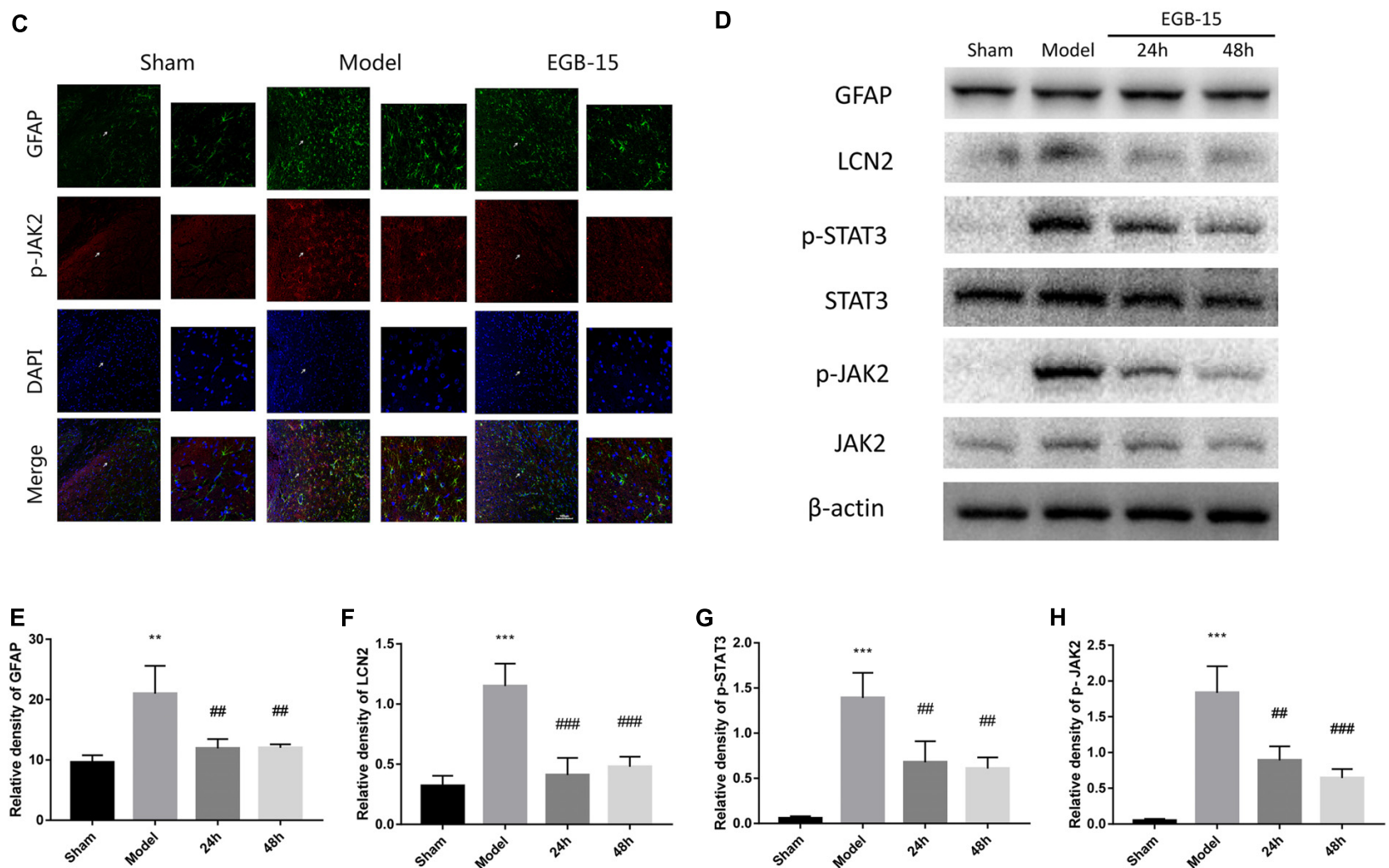

FIGURE 4 | Effects of EGB on the activation of astrocytes and the expression of LCN2, p-JAK2, p-STAT3, in cerebral ischemia rats.

(A-C) Double-immunofluorescence staining for astrocytic LCN2, p-STAT3, p-JAK2, and GFAP expression in the ischemic penumbra area after cerebral ischemia. The cells indicated with an arrow were magnified. Scale bar $=20 \mu \mathrm{m}$. (D-H) Western blots and quantitative analysis of GFAP, LCN2, p-JAK2, and p-STAT3 expression are expressed as mean $\pm \operatorname{SEM}(n=4) .{ }^{*} p<0.05,{ }^{* *} p<0.01$ and ${ }^{* * *} p<0.001$ vs. sham group; ${ }^{\#} p<0.05$, \#\# $p<0.01$ and ${ }^{\# \# \#} p<0.001$ vs. model groups.

stroke damage and protected the brain cortex from damage by decreasing infarct volume in cerebral ischemic rats. EGB ameliorated the activation of astrocytes, reduced the phosphorylation of STAT3 and JAK2 and decreased LCN2 expression in vivo and in vitro. However, LCN2 overexpression was observed to markedly reverse this result. Therefore, these results suggested that EGB exerts potent therapeutic properties in cerebral ischemia by targeting astrocytic LCN2 and suppressing neuro-inflammatory injury via the JAK2/STAT3 pathway.

Inflammation has long been known to influence the brain after cerebral ischemia, and modulating adaptive immunity may prevent the post-ischemic immune response from incurring tissue damage. Inflammatory signaling is instrumental in all stages of the ischemic cascade, from the early damaging events triggered by arterial occlusion, to the late regenerative processes 


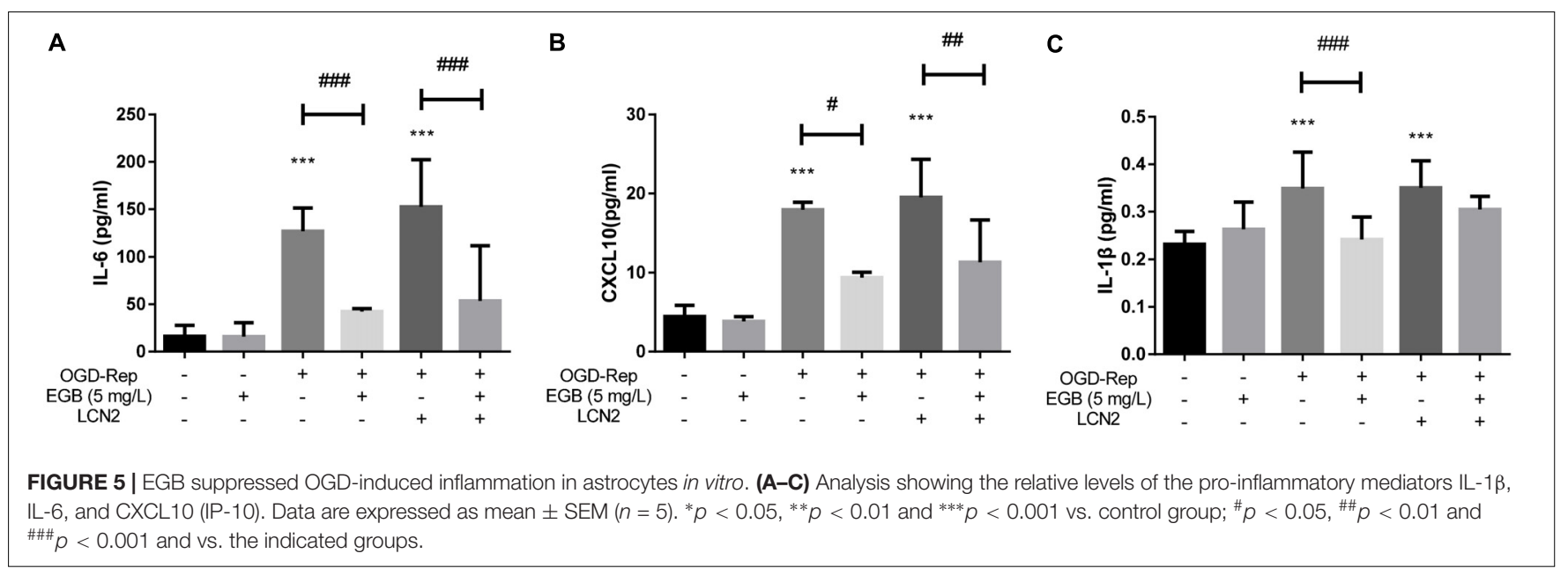

\section{A}

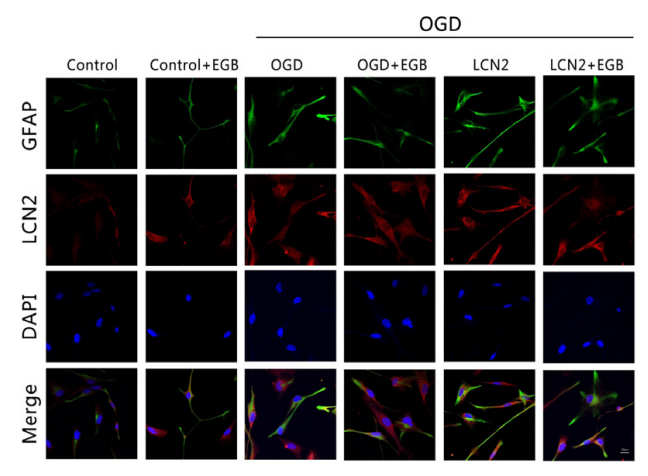

C

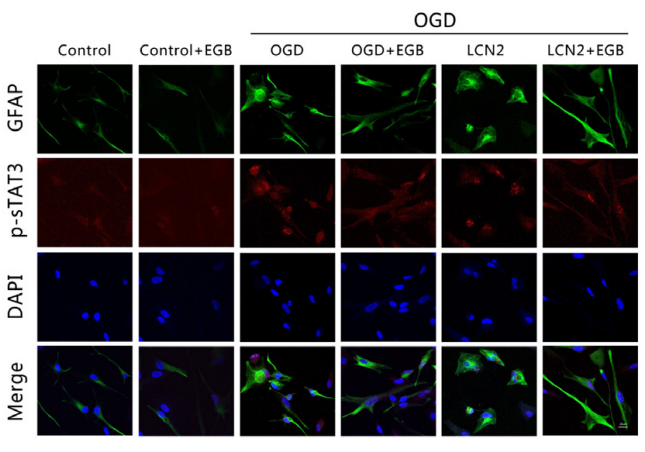

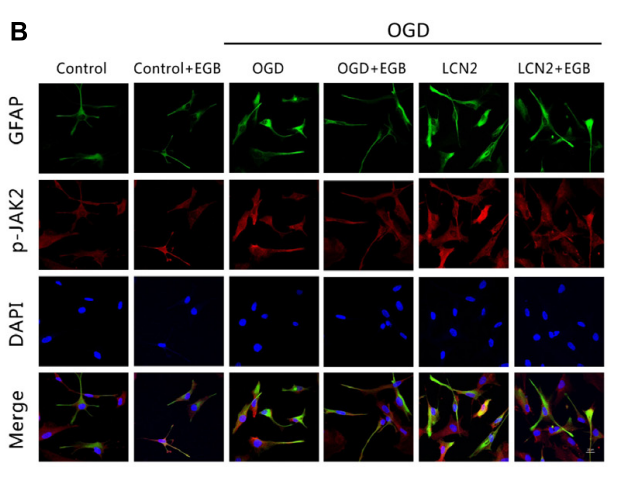

D

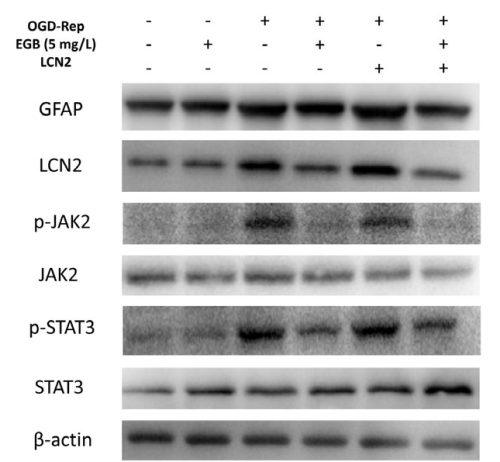

E
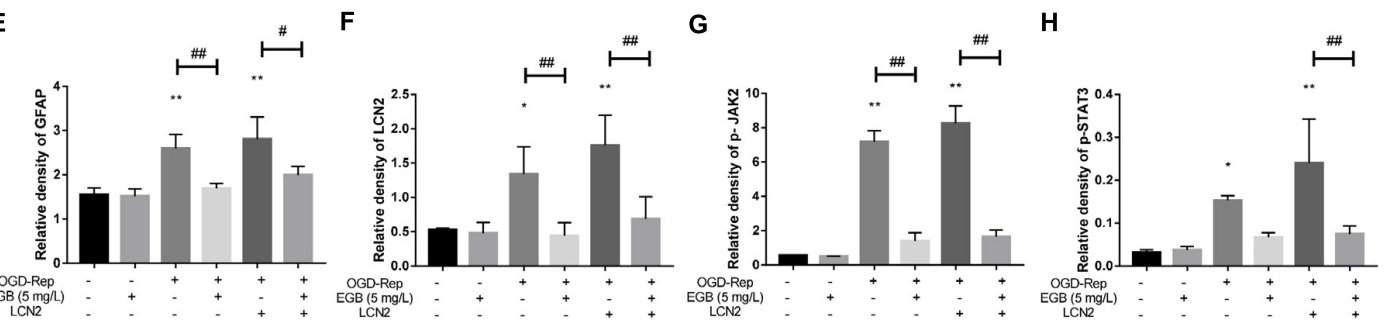

FIGURE 6 | The effects of EGB on the activation of astrocytes and the expression of astrocytic LCN2, p-JAK2, and p-STAT3 after OGD induction in vitro. (A-H) Double-immunofluorescence staining for GFAP, LCN2, p-JAK2, and p-STAT3 in astrocytes after OGD induction. Scale bar $=20 \mu \mathrm{m}$. Western blots and quantitative analysis of GFAP, LCN2, p-JAK2, and p-STAT3 expression. Data are expressed as mean \pm SEM $(n=3) .{ }^{*} p<0.05$ and $* * p<0.01$ vs. control group; ${ }^{\#} p<0.05$ and ${ }^{\# \#} p<0.01$ vs. the indicated groups. 
underlying post-ischemic tissue repair (Iadecola and Anrather, 2011). Since 1964, the effect of EGB on several disorders and diseases has been examined. Specifically, the investigated effects included (but were not limited to) cerebral insufficiency, Alzheimer's disease, POAD, and thrombosis (Maclennan et al., 2002). It has been reported that EGB, as a multifunctional neuroprotective agent, can reverse the brain oxidative damage induced by hydrogen peroxide and amyloid $\beta$-peptide, and also can suppress activation of astrocytes and microglia (Brunetti et al., 2006; Tulsulkar and Shah, 2013). In the present study, EGB was observed to reduce cerebral infarction and neuronal apoptosis in the ischemic hemisphere. Neurological disorders were also ameliorated in cerebral ischemic rats treated with EGB. Accordingly, this result provided the first evidence that EGB diminishes ischemic brain injury. The inflammatory response has both advantages and disadvantages (Fann et al., 2013), as it originally contributes to ischemic brain injury but then assists tissue regeneration (Chamorro and Hallenbeck, 2006).

The inflammatory response is characterized by a release of pro-inflammatory cytokines, including TNF- $\alpha$, IL- $1 \alpha$, IL6, and IL-18, and chemokines, including CCL2, CXCL10, and CXCL12 (Ambrosini and Aloisi, 2004). This release is mediated by neurons, astrocytes, microglia, and endothelial cells and can cause neuronal and glial cell death during cerebral ischemia (Allan and Rothwell, 2001). Indeed, it is reasonable to assume that modulating the function of pro-inflammatory cytokines and chemokines in strokes significantly affects infarct evolution (Lambertsen et al., 2012). Here, we demonstrated that EGB markedly reduced pro-inflammatory cytokines/chemokines levels, including those of IL-6, IL- $\alpha$ and CXCL10, in tissues surrounding the cerebral infarction. Additionally, EGB decreased these pro-inflammatory cytokines and chemokines in the serum.

Astrogliosis has the potential to lead to harmful effects, namely, neuroinflammation, and to interfere with synapse sprouting (Silver and Miller, 2004; Sofroniew and Vinters, 2010). Research has shown that cytokines and inflammatory responses can mediate astrocyte gene expression and physiology by impacting synaptic and neuronal functions (Sofroniew, 2014). However, the lack of effective therapeutic treatment targeting astrogliosis is becoming an issue of concern for clinical treatment. LCN2 is a member of the secreted lipocalin protein family. To date, several studies have confirmed the detrimental role of LCN2 in ischemic brains (Jin et al., 2014; Suk, 2016). In addition, several clinical trials support the experimental laboratory findings that LCN2 is a crucial component of neuroinflammation that mediates brain injury in cerebral ischemia and other pathological conditions (Chan et al., 2012; Fernandez-Cadenas et al., 2013). A recent study showed that $\mathrm{LCN} 2$ is a reactive astrocyte marker and an autocrine mediator of reactive astrogliosis (Lee et al., 2009). LCN2 amplifies neurotoxic inflammation through the activation of microglia/astrocytes and the induction of pro-inflammatory cytokines and chemokines like IL-6 and CXCL10 (Hamzic et al., 2013; Xing et al., 2014). These results suggest EGB as a new therapeutic target for suppressing innate immune responses during stroke. As demonstrated in the present study, marked astrocyte activation and LCN2 up-regulation were observed throughout the ischemic cortex area after cerebral ischemia, while EGB treatment significantly inhibited the activation of astrocytes and markedly decreased LCN2 expression. Astrocytes were also responsive to OGD-induced inflammation, as evidenced by LCN2 up-regulation. Therefore, these results provide evidence for the suppression of astrocytic LCN2 and for its contribution to the anti-inflammatory effects of EGB after cerebral ischemia occurs.

Constitutive activation of the JAK-STAT signaling pathway has been reported in cerebral ischemia. Recent investigations support a role for JAK-STAT signaling in hematopoiesis (Kisseleva et al., 2002), immune responses (Ivashkiv, 2000), cellular homeostasis (Schindler, 2002), gliogenesis (Sauvageot and Stiles, 2002), and reactive astrogliosis (Na et al., 2007; Herrmann et al., 2008). Furthermore, the activation of STAT3 by phosphorylation at Tyr705 is increased after CNS insults (Sriram et al., 2004; Yamauchi et al., 2006). STAT3 activation has been associated with increased neuroinflammation in cerebral ischemia (McGuckin et al., 2013), of which found that the JAK2-STAT3 pathway was involved in the LCN2 induction of CXCL10 secretion and possibly other phenotypic changes associated with reactive astrogliosis (Lee et al., 2011). In the present study, astrocytic p-STAT3 and p-JAK2 were upregulated after cerebral ischemia in vivo and after OGD in vitro. However, EGB acted as an effective JAK-STAT inhibitor in the cerebral ischemia model and in OGD-induced astrocytes by inducing anti-inflammatory alterations. In addition, LCN2 overexpression increased both p-STAT3 and p-JAK2 expression, but this effect was reversed by EGB treatment. These results confirmed that the JAK2/STAT3 signaling pathway is involved in LCN2-induced GFAP expression and plays a key role in LCN2 activity in astrocytes (Lee et al., 2011). Therefore, LCN2JAK2/STAT3 signaling may mediate the neuroprotective effect of EGB. Thus, we inferred that EGB suppressed neuroinflammation by inhibiting the phosphorylation of STAT3 and JAK2 in astrocytes via LCN2 suppression. However, these results do not explain all mechanisms underlying the protective effects of EGB. The alleviation of LCN2 expression was postulated as a key mechanism, as some potential indirect neuroprotective effects of EGB may still exist.

\section{CONCLUSION}

To conclude, EGB has been demonstrated to mediate neuroinflammation, which protects against ischemic brain injury by inhibiting astrogliosis. JAK2/STAT3-signaling regulation of LCN2 expression may be involved in astrogliosis and neuroinflammation. Lastly, these results imply that LCN2 could be used as a target to therapeutically modulate astrocytic responses and as an inflammatory tool for exploring the possibility of EGB as a therapeutic agent.

\section{AVAILABILITY OF DATA AND MATERIALS}

All the datasets and materials supporting the conclusions of this article are provided in the manuscript, which includes the article and the additional files. 


\section{AUTHOR CONTRIBUTIONS}

YeZ performed the research study. YoZ and JL designed the research study. BY and MY contributed to data analysis. MS, LX, CL, DC, and FT contributed to the drafting and revising of the manuscript and accepted the final manuscript.

\section{FUNDING}

This study was supported by grants of the National Basic Research Program (973 program) (China) (No. 2015CB554405).

\section{REFERENCES}

Allan, S. M., and Rothwell, N. J. (2001). Cytokines and acute neurodegeneration. Nat. Rev. Neurosci. 2, 734-744. doi: 10.1038/35094583

Ambrosini, E., and Aloisi, F. (2004). Chemokines and glial cells: a complex network in the central nervous system. Neurochem. Res. 29, 1017-1038. doi: 10.1023/B: NERE.0000021246.96864.89

Argaw, A. T., Asp, L., Zhang, J., Navrazhina, K., Pham, T., Mariani, J. N., et al. (2012). Astrocyte-derived VEGF-A drives blood-brain barrier disruption in CNS inflammatory disease. J. Clin. Invest. 122, 2454-2468. doi: 10.1172/ JCI60842

Baliutyte, G., Trumbeckaite, S., Baniene, R., Borutaite, V., and Toleikis, A. (2014). Effects of standardized extract of Ginkgo biloba leaves EGb761 on mitochondrial functions: mechanism(s) of action and dependence on the source of mitochondria and respiratory substrate. J. Bioenerg. Biomembr. 46, 493-501. doi: 10.1007/s10863-014-9590-8

Barres, B. A. (2008). The mystery and magic of glia: a perspective on their roles in health and disease. Neuron 60, 430-440. doi: 10.1016/j.neuron.2008.10.013

Bederson, J. B., Pitts, L. H., Tsuji, M., Nishimura, M. C., Davis, R. L., and Bartkowski, H. (1986). Rat middle cerebral artery occlusion: evaluation of the model and development of a neurologic examination. Stroke 17, 472-476. doi: 10.1161/01.STR.17.3.472

Brunetti, L., Orlando, G., Menghini, L., Ferrante, C., Chiavaroli, A., and Vacca, M. (2006). Ginkgo biloba leaf extract reverses amyloid beta-peptide-induced isoprostane production in rat brain in vitro. Planta Med. 72, 1296-1299. doi: $10.1055 / \mathrm{s}-2006-951688$

Chamorro, A., and Hallenbeck, J. (2006). The harms and benefits of inflammatory and immune responses in vascular disease. Stroke 37, 291-293. doi: 10.1161/01. STR.0000200561.69611.f8

Chan, C. P., Jiang, H. L., Leung, L. Y., Wan, W. M., Cheng, N. M., Ip, W. S., et al. (2012). Multiple atherosclerosis-related biomarkers associated with short- and long-term mortality after stroke. Clin. Biochem. 45, 1308-1315. doi: 10.1016/j. clinbiochem.2012.06.014

Chan, J. L., Reeves, T. M., and Phillips, L. L. (2014). Osteopontin expression in acute immune response mediates hippocampal synaptogenesis and adaptive outcome following cortical brain injury. Exp. Neurol. 261, 757-771. doi: 10.1016/j.expneurol.2014.08.015

Curtis-Prior, P., Vere, D., and Fray, P. (1999). Therapeutic value of Ginkgo biloba in reducing symptoms of decline in mental function. J. Pharm. Pharmacol. 51, 535-541. doi: 10.1211/0022357991772817

DeFeudis, F. V. (2003). A brief history of EGb 761 and its therapeutic uses. Pharmacopsychiatry 36(Suppl. 1), S2-S7. doi: 10.1055/s-2003-40450

Fann, D. Y., Lee, S. Y., Manzanero, S., Chunduri, P., Sobey, C. G., and Arumugam, T. V. (2013). Pathogenesis of acute stroke and the role of inflammasomes. Ageing Res. Rev. 12, 941-966. doi: 10.1016/j.arr.2013.09.004

Fernandez-Cadenas, I., Del Rio-Espinola, A., Domingues-Montanari, S., Mendioroz, M., Fernandez-Morales, J., Penalba, A., et al. (2013). Genes involved in hemorrhagic transformations that follow recombinant t-PA treatment in stroke patients. Pharmacogenomics 14, 495-504. doi: $10.2217 /$ pgs.13.19

Fu, Y., Liu, Q., Anrather, J., and Shi, F. D. (2015). Immune interventions in stroke. Nat. Rev. Neurol. 11, 524-535. doi: 10.1038/nrneurol.2015.144

\section{ACKNOWLEDGMENTS}

The authors are grateful to American Journal Experts for their helpful suggestions and highly qualified English language editing.

\section{SUPPLEMENTARY MATERIAL}

The Supplementary Material for this article can be found online at: https://www.frontiersin.org/articles/10.3389/fphar. 2018.00518/full\#supplementary-material

Furuya, K., Takeda, H., Azhar, S., McCarron, R. M., Chen, Y., Ruetzler, C. A., et al. (2001). Examination of several potential mechanisms for the negative outcome in a clinical stroke trial of enlimomab, a murine anti-human intercellular adhesion molecule-1 antibody: a bedside-to-bench study. Stroke 32, 2665-2674. doi: 10.1161/hs3211.098535

Hamzic, N., Blomqvist, A., and Nilsberth, C. (2013). Immune-induced expression of lipocalin-2 in brain endothelial cells: relationship with interleukin-6, cyclooxygenase-2 and the febrile response. J. Neuroendocrinol. 25, 271-280. doi: $10.1111 /$ jne. 12000

Herrmann, J. E., Imura, T., Song, B., Qi, J., Ao, Y., Nguyen, T. K., et al. (2008). STAT3 is a critical regulator of astrogliosis and scar formation after spinal cord injury. J. Neurosci. 28, 7231-7243. doi: 10.1523/JNEUROSCI.1709-08.2008

Iadecola, C., and Anrather, J. (2011). The immunology of stroke: from mechanisms to translation. Nat. Med. 17, 796-808. doi: 10.1038/nm.2399

Ivashkiv, L. B. (2000). Jak-STAT signaling pathways in cells of the immune system. Rev. Immunogenet. 2, 220-230.

Jin, M., Kim, J. H., Jang, E., Lee, Y. M., Soo Han, H., Woo, D. K., et al. (2014). Lipocalin-2 deficiency attenuates neuroinflammation and brain injury after transient middle cerebral artery occlusion in mice. J. Cereb. Blood Flow Metab. 34, 1306-1314. doi: 10.1038/jcbfm.2014.83

Kim, R. Y., Hoffman, A. S., Itoh, N., Ao, Y., Spence, R., Sofroniew, M. V., et al. (2014). Astrocyte CCL2 sustains immune cell infiltration in chronic experimental autoimmune encephalomyelitis. J. Neuroimmunol. 274, 53-61. doi: 10.1016/j.jneuroim.2014.06.009

Kisseleva, T., Bhattacharya, S., Braunstein, J., and Schindler, C. W. (2002). Signaling through the JAK/STAT pathway, recent advances and future challenges. Gene 285, 1-24. doi: 10.1016/S0378-1119(02)00398-0

Kleijnen, J., and Knipschild, P. (1992). Ginkgo biloba for cerebral insufficiency. Br. J. Clin. Pharmacol. 34, 352-358. doi: 10.1111/j.1365-2125.1992. tb05642.x

Lambertsen, K. L., Biber, K., and Finsen, B. (2012). Inflammatory cytokines in experimental and human stroke. J. Cereb. Blood Flow Metab. 32, 1677-1698. doi: $10.1038 /$ jcbfm. 2012.88

Lee, S., Kim, J. H., Kim, J. H., Seo, J. W., Han, H. S., Lee, W. H., et al. (2011). Lipocalin-2 Is a chemokine inducer in the central nervous system: role of chemokine ligand 10 (CXCL10) in lipocalin-2-induced cell migration. J. Biol. Chem. 286, 43855-43870. doi: 10.1074/jbc.M111. 299248

Lee, S., Park, J. Y., Lee, W. H., Kim, H., Park, H. C., Mori, K., et al. (2009). Lipocalin2 is an autocrine mediator of reactive astrocytosis. J. Neurosci. 29, 234-249. doi: 10.1523/JNEUROSCI.5273-08.2009

Li, Y., Zhang, Y., Wen, M., Zhang, J., Zhao, X., Zhao, Y., et al. (2017). Ginkgo biloba extract prevents acute myocardial infarction and suppresses the inflammationand apoptosis-regulating p38 mitogen-activated protein kinases, nuclear factorкB and B-cell lymphoma 2 signaling pathways. Mol. Med. Rep. 16, 3657-3663. doi: 10.3892/mmr.2017.6999

Lucas, S. M., Rothwell, N. J., and Gibson, R. M. (2006). The role of inflammation in CNS injury and disease. Br. J. Pharmacol. 147(Suppl. 1), S232-S240. doi: 10.1038/sj.bjp.0706400

Maclennan, K. M., Darlington, C. L., and Smith, P. F. (2002). The CNS effects of Ginkgo biloba extracts and ginkgolide B. Prog. Neurobiol. 67, 235-257. doi: 10.1016/S0301-0082(02)00015-1 
McGuckin, C. P., Jurga, M., Miller, A. M., Sarnowska, A., Wiedner, M., Boyle, N. T., et al. (2013). Ischemic brain injury: a consortium analysis of key factors involved in mesenchymal stem cell-mediated inflammatory reduction. Arch. Biochem. Biophys. 534, 88-97. doi: 10.1016/j.abb.2013.02.005

Mendis, S., Davis, S., and Norrving, B. (2015). Organizational update: the world health organization global status report on noncommunicable diseases 2014; one more landmark step in the combat against stroke and vascular disease. Stroke 46, e121-e122. doi: 10.1161/STROKEAHA.115.008097

Mohamed, N. E., and Abd El-Moneim, A. E. (2017). Ginkgo biloba extract alleviates oxidative stress and some neurotransmitters changes induced by aluminum chloride in rats. Nutrition 35, 93-99. doi: 10.1016/j.nut.2016.10.012

Moriyama, Y., Takagi, N., Itokawa, C., and Tanonaka, K. (2013). Injection of neural progenitor cells attenuates decrease in level of connexin 43 in brain capillaries after cerebral ischemia. Neurosci. Lett. 543, 152-156. doi: 10.1016/j.neulet.2013. 03.053

Mozaffarian, D., Benjamin, E. J., Go, A. S., Arnett, D. K., Blaha, M. J., and Cushman, M., et al. (2016). Executive summary: heart disease and stroke statistics-2016 update: a report from the American heart association. Circulation 133, 447-454. doi: 10.1161/CIR.0000000000000366

Na, Y. J., Jin, J. K., Kim, J. I., Choi, E. K., Carp, R. I., and Kim, Y. S. (2007). JAKSTAT signaling pathway mediates astrogliosis in brains of scrapie-infected mice. J. Neurochem. 103, 637-649. doi: 10.1111/j.1471-4159.2007.04769.x

Pekny, M., and Nilsson, M. (2005). Astrocyte activation and reactive gliosis. Glia 50, 427-434. doi: 10.1002/glia.20207

Pettigrew, L. C., Kindy, M. S., Scheff, S., Springer, J. E., Kryscio, R. J., Li, Y., et al. (2008). Focal cerebral ischemia in the TNFalpha-transgenic rat. J. Neuroinflammation 5:47. doi: 10.1186/1742-2094-5-47

Sauvageot, C. M., and Stiles, C. D. (2002). Molecular mechanisms controlling cortical gliogenesis. Curr. Opin. Neurobiol. 12, 244-249. doi: 10.1016/S09594388(02)00322-7

Schindler, C. W. (2002). Series introduction. JAK-STAT signaling in human disease. J. Clin. Invest. 109, 1133-1137. doi: 10.1172/JCI0215644

Silver, J., and Miller, J. H. (2004). Regeneration beyond the glial scar. Nat. Rev. Neurosci. 5, 146-156. doi: 10.1038/nrn1326

Sofroniew, M. V. (2009). Molecular dissection of reactive astrogliosis and glial scar formation. Trends Neurosci. 32, 638-647. doi: 10.1016/j.tins.2009.08.002

Sofroniew, M. V. (2014). Multiple roles for astrocytes as effectors of cytokines and inflammatory mediators. Neuroscientist 20, 160-172. doi: 10.1177/ 1073858413504466

Sofroniew, M. V., and Vinters, H. V. (2010). Astrocytes: biology and pathology. Acta Neuropathol. 119, 7-35. doi: 10.1007/s00401-009-0619-8

Sriram, K., Benkovic, S. A., Hebert, M. A., Miller, D. B., and O'Callaghan, J. P. (2004). Induction of gp130-related cytokines and activation of JAK2/STAT3 pathway in astrocytes precedes up-regulation of glial fibrillary acidic protein in the 1-methyl-4-phenyl-1,2,3,6-tetrahydropyridine model of neurodegeneration: key signaling pathway for astrogliosis in vivo. J. Biol. Chem 279, 19936-19947. doi: 10.1074/jbc.M309304200

Suk, K. (2016). Lipocalin-2 as a therapeutic target for brain injury: an astrocentric perspective. Prog. Neurobiol. 144, 158-172. doi: 10.1016/j.pneurobio.2016. 08.001

Tulsulkar, J., and Shah, Z. A. (2013). Ginkgo biloba prevents transient global ischemia-induced delayed hippocampal neuronal death through antioxidant and anti-inflammatory mechanism. Neurochem. Int. 62, 189-197. doi: 10.1016/ j.neuint.2012.11.017

Wang, Y., Wang, R., Wang, Y., Peng, R., Wu, Y., and Yuan, Y. (2015). Ginkgo biloba extract mitigates liver fibrosis and apoptosis by regulating p38 MAPK,

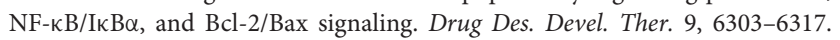
doi: 10.2147/DDDT.S93732

Xing, C., Wang, X., Cheng, C., Montaner, J., Mandeville, E., Leung, W., et al. (2014). Neuronal production of lipocalin-2 as a help-me signal for glial activation. Stroke 45, 2085-2092. doi: 10.1161/STROKEAHA.114. 005733

Yamauchi, K., Osuka, K., Takayasu, M., Usuda, N., Nakazawa, A., Nakahara, N., et al. (2006). Activation of JAK/STAT signalling in neurons following spinal cord injury in mice. J. Neurochem. 96, 1060-1070. doi: 10.1111/j.1471-4159. 2005.03559.x

Zamanian, J. L., Xu, L., Foo, L. C., Nouri, N., Zhou, L., Giffard, R. G., et al. (2012). Genomic analysis of reactive astrogliosis. J. Neurosci. 32, 6391-6410. doi: 10.1523/JNEUROSCI.6221-11.2012

Zhang, R. L., Chopp, M., Li, Y., Zaloga, C., Jiang, N., Jones, M. L., et al. (1994). AntiICAM-1 antibody reduces ischemic cell damage after transient middle cerebral artery occlusion in the rat. Neurology 44, 1747-1751. doi: 10.1212/WNL.44.9. 1747

Zhang, Y., Miao, L., Lin, L., Ren, C. Y., Liu, J. X., and Cui, Y. M. (2018). Repeated administration of Sailuotong, a fixed combination of Panax ginseng, Ginkgo biloba, and Crocus sativus extracts for vascular dementia, alters CYP450 activities in rats. Phytomedicine 38, 125-134. doi: 10.1016/j.phymed.2017. 02.007

Conflict of Interest Statement: The authors declare that the research was conducted in the absence of any commercial or financial relationships that could be construed as a potential conflict of interest.

Copyright (c) 2018 Zhang, Liu, Yang, Zheng, Yao, Sun, Xu, Lin, Chang and Tian. This is an open-access article distributed under the terms of the Creative Commons Attribution License (CC BY). The use, distribution or reproduction in other forums is permitted, provided the original author(s) and the copyright owner are credited and that the original publication in this journal is cited, in accordance with accepted academic practice. No use, distribution or reproduction is permitted which does not comply with these terms. 